This item was submitted to Loughborough's Research Repository by the author.

Items in Figshare are protected by copyright, with all rights reserved, unless otherwise indicated.

\title{
Consideration of IEEE 802.11p and proposed 5G for holograms in vehicular communication
}

PLEASE CITE THE PUBLISHED VERSION

https://doi.org/10.1049/cp.2018.1245

PUBLISHER

(C) IET

VERSION

AM (Accepted Manuscript)

\section{PUBLISHER STATEMENT}

This work is made available according to the conditions of the Creative Commons Attribution-NonCommercialNoDerivatives 4.0 International (CC BY-NC-ND 4.0) licence. Full details of this licence are available at: https://creativecommons.org/licenses/by-nc-nd/4.0/

\section{LICENCE}

CC BY-NC-ND 4.0

\section{REPOSITORY RECORD}

Thomas, M., R.M. Edwards, and Zhe Wang. 2019. "Consideration of IEEE 802.11p and Proposed 5G for Holograms in Vehicular Communication”. figshare. https://hdl.handle.net/2134/36592. 


\title{
Consideration of IEEE 802.11p and proposed 5G for Holograms in Vehicular Communication.
}

\author{
M. Thomas ${ }^{1}$, R. M. Edwards ${ }^{1}$ and Z. Wang ${ }^{1}$ \\ ${ }^{1}$ 5G Research Centre, Electronic Engineering, Loughborough University, UK, r.m.edwards@lboro.ac.uk
}

\begin{abstract}
Vehicular communication is the technology that allows vehicles to exchange information with other cars and its surroundings to enhance safety and efficiency of transportation systems. Informative communication, which includes vehicle's position, velocity, and location, enables the sensing of hazards and traffic congestion. In this paper, two vehicular communication standards, Institute of Electrical and Electronics Engineers (IEEE) 802.11p and the proposed nextgeneration cellular network 5G are compared for vehicular networking. A detailed comparative study of the standards concerning latency, coverage, scalability, and mobility. The results indicate that IEEE $802.11 \mathrm{p}$ offers acceptable performance with limited mobility support. Whereas, 5G meets most of the vehicular application requirements regarding latency, coverage, scalability, and mobility. 3D holographic communication in 5G would allow users to experience live and interactive meetings. The bandwidth requirement of $3 \mathrm{D}$ holograms is predicted to be in terabyte level. With compression techniques, the delivery of real-time holograms has been researched to require $10 \mathrm{Gbps}$ or higher.
\end{abstract}

Index Terms-DSRC, 802.11p 5G, D2D, Hologram, 3D Graphics.

\section{INTRODUCTION}

Vehicular Communication or Vehicle-to-Everything (V2X) communication is an emerging communication technology for road safety, traffic efficiency, fuel efficiency, and infotainment. V2X communication includes the Vehicleto-Vehicle (V2V), Vehicle-to-Infrastructure (V2I), Vehicleto-Pedestrian (V2P), Vehicle-to-Device (V2D) and Vehicleto-Network (V2N) Communication. Various communication standards like Dedicated Short-Range Communication (DSRC) in the US, Intelligent Transportation Systems (ITS) - G5 of the European Telecommunication Standards Institute (ETSI) in the Europe, are developed across the world. DSRC and ITS-G5 are based on the Wireless Access in Vehicular Environment (WAVE) Standards. WAVE protocol stack constitutes of IEEE 802.11p and IEEE 1609 standards. The short communication latency of WAVE standards makes it suitable for V2X communication. Some of the problems faced by this Wi-Fi-based communication are low reliability, channel access delay, low mobility and lack of quality-ofservice (QoS) guarantee. The current generation, 4G, and the next generation, 5G, cellular network systems have been considered by many authors as a possible as a solution for vehicular communication. Cellular networks have base station infrastructure which provides large coverage, reduced latency, and high reliability. In fact, the 3 GPP requirements for $5 \mathrm{G}$ include targets that go beyond all the specifications for the current iteration of $802.11 \mathrm{p}$ that were specifically designed for V2X. However, 802.11p is ready now and may be unlicensed therefore adoption may be rapid whilst ultrareliable, low latency facets of the proposed $5 \mathrm{G}$ are required by autonomous vehicles and may not become available for five years or more.

In this paper, we have assumed that the $5 \mathrm{G}$ requirements will be substantially achieved and that $3 \mathrm{D}$ Graphics, also known as Holograms, will be the killer app for the new communications system.

In 1947, Dennis Gabor coined the terms hologram and holography. 'Holos' in Greek means whole, complete and 'gramma' means a letter, writing. A hologram is a recording of the interference pattern, in a two-dimensional or threedimensional medium, formed when a point source of light, named reference beam, of a particular wavelength, confronts light of the same wavelength received from an object (object beam). If the hologram is illuminated by the reference beam alone, the diffraction pattern reconstructs the wave fronts of light from the original object. The process of creating a hologram is called holography. Holographic transmission requires high data rates and less latency. At present, these high data rates could only be satisfied in 3GPP proposed $5 \mathrm{G}$ but not in $802.11 \mathrm{p}$. Holograms infotainment are obvious how will occupants of driverless cars occupy themselves but well before that Holograms could be used for 3D medical imaging by medics on the way to accident, planning by services on their way to a fire and strategy by law enforcement on their way to an incident.

The rest of this paper is organized as follows. Section II describes the related work. An overview of the IEEE $802.11 \mathrm{p}$ standard in the vehicular networking domain is given in Section III. In Section IV, an overview of the $5 \mathrm{G}$ standard in the vehicular networking is explained. The comparison of IEEE 802.11p and 5G is done in Section V. In Section VI, holographic transmission is explained. Finally, the paper is concluded in Section VII.

\section{RELATED WORK}

The exchange of information between vehicles, infrastructure and pedestrians have become important in vehicular communication. Many works have been done for the enhancements of vehicular communication standards like IEEE $802.11 \mathrm{p}$ and $5 \mathrm{G}$.

In the analytical modelling of IEEE $802.11 p$ [1-4], the authors investigated the delay with traffic and networking 
conditions, throughput, collision rate, coverage. A comparison between IEEE $802.11 \mathrm{p}$ and WiMAX technology and the impact of data rate and vehicle speed are measured for both standards in [5]. M. Gholibeigi et al. introduced a single-hop receiver-based reliable broadcasting variation to show how messages can be distributed reliably in a network of nodes using IEEE 802.11p [6]. In [7] G. Remy et al. used a Multi-Hop forwarding communication system in aggregation with cellular technology. The author discussed the Decentralized Congestion Control (DCC) algorithm to deal with the increased network load of using a multi-hop enhancement of IEEE 802.11p [8].

According to the METIS project [9-10], foundation for the 5G, ultra-reliable Machine Type Communication (uMTC) targets at low latency high reliable communication. The works of METIS project includes the requirements and test cases for 5G. E.G. Strom et al. [11] discuss the uMTC for vehicular communication compared to IEEE 802.11p. Also, the integration approaches between IEEE 802.11p and uMTC are examined. The concept of Device-to-Device communication (D2D), for high reliability and high data rates in vehicular communication, is mentioned by W. Sun et al in [12]. In [13] the authors found that the applicability of LTE with the centralized base station is challenging for rapidly moving V2V communication. The authors of [14] concluded that LTE performs significantly better than IEEE $802.11 \mathrm{p}$ in the vehicular communication.

Ponchitra et al calculated the memory size for different pixel sizes. A $512 \times 512 \times 3$ hologram occupies a memory space of $42.8 \mathrm{~KB}$ whereas, for $256 \times 256 \times 3$ pixels, memory space of $15.9 \mathrm{~KB}$ is used. For an input video file of $30.9 \mathrm{MB}$, $140 \times 180 \times 3$ of hologram video requires $11.3 \mathrm{MB}$. In the transmission of image/video, edges are more sensitive to noise compared to other low-frequency components. The time required to transmit $512 \mathrm{Kbps}$ of a video file without compression is 506.27 seconds and after compression, it would be 185.14 seconds [15].

Xuewu $\mathrm{Xu}$ [16] et al computed the total data bandwidth of a holographic video to be $9.44 \mathrm{Gbps}$ at 60 object frames per second (1440 binary holograms per second). The hologram data packages transmitted using TCP/IP data stream via the network can be reliably played back as a holographic video at $60 \mathrm{fps}$ with the display system developed. Hologram data compression and 3D object data transmission are two potential solutions to reduce the bandwidth requirement for practical holographic video transmission over the network.

5G technology should allow users to enjoy realistic services such as augmented/virtual reality and holograms. As holography requires ultra-high volume data transmission, an ultra-high speed real-time data processing technology infrastructure and hologram-based $\mathrm{I} / \mathrm{O}$ equipment is needed. On an average, a compressed 3D hologram with a pixel resolution of $1920 * 1080 * 50$ requires $200 \mathrm{MB}$. Therefore, an uncompressed $3 \mathrm{D}$ hologram requires terabyte bandwidth [17].

\section{VEHICULAR COMMUNICATION USING IEEE 802.11P}

The WAVE standard constitutes IEEE $802.11 \mathrm{p}$ for Physical \& MAC layers and IEEE 1609 for security, network management. In 1999, the U.S. Federal Communications Commission (FCC) set aside $75 \mathrm{MHz}$ of bandwidth, in the $5.9 \mathrm{GHz}$ region $(5.850$ - 5.925) $\mathrm{GHz}$, for $\mathrm{V} 2 \mathrm{x}$ communication. The two Working Groups (WG) of Institute of Electrical and Electronics Engineers (IEEE) involved in the development of DSRC standards are 1609 WG and 802.11p WG. IEEE 1609 WG concentrated on standards from higher Medium Access Control (MAC) layer to application layers, whereas, IEEE 802.11p focused on lower MAC and Physical (PHY) layer. The details of IEEE 1609 standard are as follows. IEEE 1609.2 standard is accountable for the communication security. IEEE 1609.3 standard includes the WAVE connection setup and management. IEEE 1609.4 standard is responsible for higher layers of MAC layer.

The properties of IEEE $802.11 \mathrm{p}$ PHY standard is inherited from IEEE 802.11a. The PHY of the IEEE 802.11p is composed of two sub layers Physical Layer Convergence Protocol (PLCP) which is responsible for communicating with the MAC layer which alters the Packet Data Unit (PDU) arriving from the MAC layer to make up an OFDM frame; and Physical Medium Access (PMD) is the interface to the physical transmission medium (radio channels and fibre links) and manage data encoding and perform the modulation. The $75 \mathrm{MHz}$ bandwidth, in the $5.9 \mathrm{GHz}$ region, is divided into seven $10 \mathrm{MHz}$ channels. The initial $5 \mathrm{MHz}$ is reserved for Guard Band (GB). Channels 1, 2, 3, 5, 6, 7 are known as service channels. And channel 4 is known as control channel which is allocated for low latency service messages. For higher throughput, channels can be merged and used as a channel with a maximum channel bandwidth of up to $20 \mathrm{MHz}$. In the V2X communication, the moving transmitter and receiver, stationary objects, causes multipath fading of signals at the receiver. Therefore, the bit rate is reduced for each channel using Orthogonal Frequency Division Multiplexing (OFDM) to handle inter-carrier interference caused by Doppler spread due to moving vehicles. The IEEE $802.11 \mathrm{p}$ can support data transmission rates ranging from 3 to $27 \mathrm{Mbit} / \mathrm{s}$, differing on the modulation and the puncturing schemes. In IEEE 802.11p transmitter, for forward error correction, a convolutional encoder is employed at the beginning. The coded data is interleaved to mitigate burst errors caused by impulse noises. A 64-point inverse Fast Fourier Transform (IFFT) implements the OFDM modulation. The 64 OFDM subcarriers include 48 data subcarriers and 4 phase tracking pilot subcarriers.

IEEE 802.11e standard defines MAC sub layer procedures for IEEE 802.11p MAC layer. IEEE 802.11 protocol defines the Basic Service Set (BSS), which represents a group of stations and an Access Point (AP) to communicate with each other over the air interface. The devices in IEEE 802.11 are required to be a member of a $\mathrm{BSS}$ to exchange messages. For V2X communication, 
vehicles in communication range need to exchange data immediately, without prior exchange of control information. Outside the context of a BSS (OCB) disables all control procedures common in BSS for the V2X communication. Moreover, when a station supports several modes, it can be configured to a single mode at a time only, i.e. OCB or infrastructure mode. The stations in OCB mode use the enhanced distributed channel access (EDCA) for medium access. EDCA employs the Carrier Sense Multiple Access with Collision Avoidance (CSMA/CA) as it is contentionbased. With CSMA/CA, a device pays attention to the channel before it starts its own transmission. If the channel is engaged, the station delays its transmission by a random duration of time. Stations distinguish data by assigning the data to access categories (ACs) and handling the data from different access categories with other CSMA/CA-relate parameters, which will effectively allow for data traffic prioritization. The absence of infrastructure for channel access for high-speed vehicles possesses significant challenge in terms of network performance.

\section{VEHICULAR COMMUNICATION USING 5G}

Different research groups like Mobile and Wireless Communications Enablers for the Twenty-twenty Information Society (METIS), Enhanced Multicarrier Technology for Professional Ad-Hoc and Cell-Based Communications (EMPhAtiC), 5G Infrastructure Public Private Partnership (5GPPP), 5G Innovation Centre at the University of Surrey, NYU WIRELESS, and the Electronics and Telecommunications Research Institute (ETRI), Korea are working on $5 \mathrm{G}$ standards. METIS has identified that $5 \mathrm{G}$ focuses on providing very high data rates with very low latency, commendable user experiences in densely populated areas, vehicular communication, reliability and efficient management of a large number devices.

Device-to-Device (D2D) Communication is expected to play a crucial role in the approaching era of $5 \mathrm{G}$ with enhanced spectral efficiency and system capacity and reduced latency within the networks. In the D2D communication, the Mobile Network Operator (MNO) does not interfere with the transmission from source to destination and the D2D links provide direct communication between surrounding devices and offload the traffic of MNOs. D2D can operate in limited or no network connectivity. Therefore, the broadcast communication between nearby vehicles is possible in remote areas with less cellular coverage.

$5 \mathrm{G}$ services like Enhanced Mobile Broad-Band (xMBB), Massive Machine-Type Communication (mMTC), Ultrareliable Machine-Type Communication (uMTC) helps in vehicular communication. uMTC provides ultra-reliable low latency communication network mainly for V2I communication. mMTC provide wireless connectivity for many devices which can be utilized for $\mathrm{V} 2 \mathrm{~V}$ communication. xMBB provides high data rates and good Quality of Experience (QoE) which can be utilised by passengers for entertainment purposes like downloading and uploading data. Even though enhancements of current generation cellular networks are being done, 5G with high reliability and low latency is proposed to be the perfect solution for vehicular communication.

\section{COMPARISON BETWEEN IEEE 802.11P AND 5G}

A brief comparison of IEEE $802.11 \mathrm{p}$ and $5 \mathrm{G}$ is as follows.

\section{A. Licensed Spectrum}

Most 5G will exist in licensed spectrum whereas there are currently no proposals to licence the IEEE $802.11 \mathrm{p}$ spectrum. The advantage of a licensed spectrum is that good QoS is provided. With centralized channel management in the given frequencies and bands, the devices will have a guaranteed QoS without interference. As there is no restriction for transmit power, more devices can be accessed even in limited connectivity areas. $5 \mathrm{G}$ is proposed to have a diverse set of spectrum bands at lower frequencies for enabling wide area coverage, at higher frequencies with large bandwidths, to support a very high number of devices, at very high frequencies above $24 \mathrm{GHz}$ for providing ultra-high capacity and very low latency.

\section{B. Coverage}

The deployment of base station infrastructure solves the problem of short lived connectivity of IEEE $802.11 \mathrm{p}$. In urban areas, due to large buildings, signals fade out in NonLine of Sight (NLOS) propagation paths of IEEE 802.11p. Base stations have a coverage of $1000 \mathrm{~m}$ and are located at higher positions. Therefore, $5 \mathrm{G}$ provides better performance in NLOS environments. D2D links can operate with limited or no LOS connectivity. In limited connectivity, slowly varying Channel State Information (CSI) or slowly varying context information is used for Radio Resource Management (RRM). Whereas in no connectivity, Self-organized Time Division Multiple Access(STDMA) or code slotted Aloha are used.

\section{Scalability}

5G has a large bandwidth relative to IEEE 802.11p and this tends to access by a larger number of devices. In broadcast communication, IEEE802.11p lacks a mechanism to broadcast messages to a large range of devices. D2D links of $5 \mathrm{G}$ should support large numbers of nodes. 5G D2D can support vehicles with speeds up to $350 \mathrm{~km} / \mathrm{hr}$ whereas $802.11 \mathrm{p}$.

\section{Cost-CAPEX/OPEX}

The use of base stations in $5 \mathrm{G}$ reduces the installation and maintenance cost as one base station can support devices in the range of $1000 \mathrm{~m}$. Whereas in IEEE 802.11p, a large number of Road-Side Units (RSU) for coverage and communication hence the CAPEX/OPEX increases. 


\section{E. Capacity}

$5 \mathrm{G}$ is targeted to offer at least $20 \mathrm{Gbps}$ in downlink and $10 \mathrm{Gbps}$ in uplink. With the use of efficient modulation schemes and antenna techniques, the data rates can be increased and a large number of devices can be supported in a cell. The IEEE $802.11 \mathrm{p}$ can support data transmission rates ranging from 3 to $27 \mathrm{Mbit} / \mathrm{s}$, differing on the modulation and the puncturing schemes.

\section{F. Infotainment}

$5 \mathrm{G}$ with high data rate and large bandwidth provide highquality audio and video communications. With D2D and uMTC, future vehicles can exchange information such as various sensor data, emergency warning messages. Passengers can be provided with high-quality audio and video streaming, email updates, software updates etc. In IEEE $802.11 \mathrm{p}$, due to low data rates, the essential vehicle data are transmitted and entertainment is not supported.

\section{G. Latency}

IEEE 802.11p MAC layer CSMA/CA causes a delay in channel access. A latency of more than $60 \mathrm{~ms}$ occurs in IEEE $802.11 \mathrm{p}$. Whereas in $5 \mathrm{G}$, METIS project proposed a latency of $1 \mathrm{~ms}$ with $99.9 \%$ reliability. This reduced latency helps in the transmission of emergency traffic warning messages. The uMTC along with D2D communication can inform the vehicles the status of the network and thereby the network traffic can be offloaded where the vehicles can choose their services accordingly. D2D communication is possible using Proximity Service (ProSe) or by direct base station infrastructure.

\section{HOLOGRAPHIC TRANSMISSION USING 5G}

Holographic Communication is a new field of research in which the sender and receiver can use three-dimensional (3D) imaging. Holograms are of terabyte bandwidth. Using Compression techniques like scalar and vector quantization, Discrete Cosine Transform (DCT) and wavelet-based transform, the bandwidth of the holograms can be reduced to megabyte or gigabyte level. Holograms with data rates up to $10 \mathrm{Gbps}$ can be transmitted in $5 \mathrm{G}$. $5 \mathrm{G}$ is expected to provide a data rate of 10 to $50 \mathrm{Gbps}$. Researches are being done on the holographic communication system devices. With the development of holographic communication technology, users can attend meetings even without being at the venue. Also, the present view of the road or place can help the users in vehicular communication.

\section{CONCLUSION}

A comparative study of IEEE $802.11 \mathrm{p}$ and $5 \mathrm{G}$ for vehicular networking has been done in this paper. The standards were evaluated in terms of coverage, delay, reliability, scalability, and mobility. Thus, it can be concluded that the next generation cellular network $5 \mathrm{G}$ is suitable for most of the V2X communication. IEEE 802.11p standard offers low mobility transmission in the $5.9 \mathrm{GHz}$ transmission range. Depending on the vehicle speed, traffic load and traffic density, 5G may outperform IEEE 802.11p. With the latency of less than $100 \mathrm{~ms}$ and data rate up to 27 Mbps, IEEE 802.11p can be used in V2X services. Along with 5G research, holographic communication is a significant research topic in the future. At the Mobile World Congress (MWC 2017) Barcelona, the operator Ericsson presented a beamforming V2X system with a car travelling at $170 \mathrm{~km} /$ hour and demonstrated a speed of $3.6 \mathrm{Gbps}$. The test network was operating in the $28 \mathrm{GHz}$ band. Ericsson also claimed throughput above $1.5 \mathrm{Gbps}$ for data transmission while switching. In 2016 another operator, Huawei claimed 11.29 Gbps with $<0.5 \mathrm{mS}$ latency for 23 simultaneously connected devices. However, as yet there are no implementations of $5 \mathrm{G}$. Combined with there are several holographic technologies, both stereoscopic (with glasses) and auto-sterioscopic (glasses free) appearing on the market. The streaming 3D colour holographic video of a dancing bear at $60 \mathrm{fps}$ over a network has been successfully demonstrated by the authors of [16] .

\section{REFERENCES}

[1] KA Hafeez, L Zhao, Z Liao, BN Ma, Performance analysis of broadcast messages in VANETs safety applications, in Proceedings of the IEEE Global Telecommunications Conf. GLOBECOM 2010, Miami, FL, 6-10 December 2010

[2] C Han, M Dianati, R Tafazolli, R Kernchen, Throughput analysis of the IEEE 802.11p enhanced distributed channel access function in vehicular environment, in Proceedings of the IEEE 72nd Vehicular Technology Conference Fall (VTC 2010-Fall), (Ottawa, ON). 6-9 September 2010

[3] R Gallardo, D Makrakis, HT Mouftah, Performance analysis of the EDCA medium access mechanism over the control channel of an IEEE 802.11p WAVE vehicular network, in Proceedings of the IEEE International Conference on Communications (ICC 2009) (Dresden). 14-18 June 2009

[4] J Misic, G Badawy, S Rashwand, VB Misic, Tradeoff issues for $\mathrm{CCH} / \mathrm{SCH}$ duty cycle for IEEE $802.11 \mathrm{p}$ single channel devices, in Proceedings of the IEEE Global Telecommunications Conference GLOBECOM 2010 (Miami, FL). 6-10 December 2010

[5] IC Msadaa, P Cataldi, F Filali, Comparative study between $802.11 \mathrm{p}$ and mobile WiMAX-based V2I communication networks, in Proceedings of the Fourth International Conference on Next Generation Mobile Applications, Services and Technologies (NGMAST), (Amman). 27-29 July 2010

[6] M. Gholibeigi, G. Heijenk, D. Moltchanov, Y. Koucheryavy, Analysis of a Receiver-based Reliable Broadcast Approach for Vehicular Networks. 2014 IEEE Vehicular Networking Conference (VNC), Paderborn, 2014, pp. 89-96.

[7] G. Remy et al., "LTE4V2X-Collection, Dissemination and Multi-Hop Forwarding," IEEE ICC, June 2012

[8] 5G-PPP, 5G Automotive Vision, October 2015

[9] MET15-D43 ICT-317669 METIS, Deliverable 1.1 Version 1 "Scenarios, requirements and KPIs for 5G mobile and wireless system", April 2014

[10] MET15-D43 ICT-317669 METIS, Deliverable 4.3 Version 1 "Final report on network level solutions", February 2015

[11] E.G. Ström, P. Popovski, J. Sachs 1993. 5G Ultra-Reliable Vehicular Communication. 2015

[12] W. Sun, E. G. Ström, F. Brännström, Y. Sui and K. C. Sou, "D2Dbased V2V communications with latency and reliability constraints," 
2014 IEEE Globecom Workshops (GC Wkshps), Austin, TX, 2014, pp. 1414-1419

[13] G. Araniti, C. Campolo, M. Condoluci, A. Iera and A. Molinaro, "LTE for vehicular networking: a survey," in IEEE Communications Magazine, vol. 51, no. 5, pp. 148-157, May 2013

[14] Z. Hameed Mir and F. Filali, "LTE and IEEE 802.11p for vehicular networking: a performance evaluation" in EURASIP Journal on Wireless Communications and Networking, no.1, pp.89

[15] S.Ponchitra, K.Murali, P.K.Palanisamy, "Digital Video HolographyA Robust Tool for Communication”, pp. 307-316, 2012.

[16] Xuewu Xu, Yuechao Pan, Phyu Phyu Mar Yi Lwin, Xinan Liang, "3D Holographic Display and Its Data Transmission Requirement", IPOC 2011, pp. 1-4.

[17] SK Telecom 5G white Paper. 